\title{
A Note on Derivative of Sine Series with Square Root
}

\author{
Sergiusz Kęska \\ Faculty of Exact and Natural Sciences, Siedlce University of Natural Sciences and Humanities, Poland \\ Correspondence should be addressed to Sergiusz Kęska; keska@se.onet.pl
}

Received 6 September 2021; Accepted 8 October 2021; Published 8 November 2021

Academic Editor: Victor Kovtunenko

Copyright (c) 2021 Sergiusz Kęska. This is an open access article distributed under the Creative Commons Attribution License, which permits unrestricted use, distribution, and reproduction in any medium, provided the original work is properly cited.

Chaundy and Jolliffe proved that if $\left\{a_{n}\right\}$ is a nonnegative, nonincreasing real sequence, then series $\sum a_{n} \sin (n x)$ converges uniformly if and only if $n a_{n} \longrightarrow 0$. The purpose of this paper is to show that if $\left\{n a_{n}\right\}$ is nonincreasing and $n a_{n} \longrightarrow 0$, then the series $f(x)=\sum a_{n} \sin (\sqrt{n} x)$ can be differentiated term-by-term on $[c, d]$ for $c, d>0$. However, $f^{\prime}(0)$ may not exist.

\section{Introduction}

Chaundy and Jolliffe [1] proved the following.

Theorem 1. If $\left\{c_{k}\right\}_{k=1}^{\infty} \subset R_{+}$is decreasing to zero, then $\sum_{k=1}^{\infty}$ $c_{k} \sin (k x)$ converges uniformly in $x$ if and only if $k c_{k} \longrightarrow 0$ as $k \longrightarrow \infty$.

Theorem 1 has had numerous generalizations.

Leindler [7] verified that in Theorem 1, the monotonicity assumption $c_{n} \geq c_{n+1}$ can be replaced by $c \in$ RBVS, i.e., if the conditions $c_{n} \longrightarrow 0$ and $\sum_{k=n}^{\infty}\left|c_{k}-c_{k+1}\right| \leq K c_{n}$ hold for all $n$ with constant $K=K(c)$ which depends only upon $c$.

The next theorem was indicated in [11].

Theorem 2. If $\left\{c_{k}\right\}$ belongs to the class MVBVS, i.e., if there exist constants $C$ and $\lambda \geq 2$, depending only on the sequence $\left\{c_{k}\right\}$ such that $\sum_{k=n}^{2 n}\left|c_{k}-c_{k+1}\right| \leq(C / n) \sum_{k=\left\lfloor\lambda^{-1} n\right\rfloor}^{\lfloor\lambda n} c_{k}$ for all $n \geq \lambda$, then series $\sum_{k=1}^{\infty} c_{k} \sin (k x)$ converges uniformly in $x$ if and only if $\lim _{k \rightarrow \infty} k c_{k}=0$.

Móricz [8] proves the following theorem.

Theorem 3. Assume $f: R_{+} \longrightarrow[0, \infty)$ with property $x f(x)$ $\in L_{\text {loc }}^{1}\left(R_{+}\right)$. If $f(x)$ is nonincreasing on $R_{+}$, then the integral $\int_{0}^{\infty} f(x) \sin (t x) d x, t \in R_{+}$, converges uniformly in $t$ if and only if $x f(x) \longrightarrow 0$ as $x \longrightarrow \infty$.
A result due to Žak and Šneider [10] holds for double sine series.

Theorem 4. If $\left\{c_{j k}\right\}_{j, k=1}^{\infty} \subset R_{+}$is monotonically decreasing double sequences, i.e., a sequence of real numbers such that for $j, k=1,2, \cdots, \quad c_{j k}-c_{j+1, k} \geq 0, \quad c_{j k}-c_{j, k+1} \geq 0$, and $c_{j k}-$ $c_{j, k+1}-c_{j+1, k}+c_{j+1, k+1} \geq 0$, then $\sum_{j=1}^{\infty} \sum_{k=1}^{\infty} c_{j k} \sin (j x) \sin (k y)$ is uniformly regularly convergent in $(x, y)$ if and only if $j k$ $c_{j k} \longrightarrow 0$ as $j+k \longrightarrow \infty$.

Theorem 4 was generalized by Kórus [6]. He has defined new classes of double sequences $\left(\right.$ SBVDS $_{1}$ ) to obtain those generalizations.

Duzinkiewicz and Szal [2] introduce a new class of double sequences called $\operatorname{DGM}(\alpha, \beta, \gamma, r)$, which is a generalization of the class considered by Kórus, and they obtain sufficient and necessary conditions for uniform convergence of double sine series.

Dyachenko et al. [3] proved the Chaundy-Jolliffe theorem for $\operatorname{GM}(\beta)$ sequences with majorant $\beta$ having the following form: $\beta_{n}=(1 / n) F_{n}(a)$, where $F_{n}$ is admissible.

In the recent paper [5], it was proved that $\sum a_{n} \sin (\sqrt{n} x)$ converges uniformly on $[0, \pi]$ if and only if $n a_{n} \longrightarrow 0$.

One of the results of paper [9] is that for any $\alpha \in(0,2)$, $\sum a_{n} \sin n^{\alpha} x$ converges uniformly if and only if $n a_{n} \longrightarrow 0$. 


\section{Main Results}

Lemma 5. Let

$$
* \cos \left(\frac{\pi}{2 x_{0}}\left(\sqrt{\left\lfloor\frac{x_{0}^{2}(m+1)^{2}}{4}-j\right\rfloor}+\sqrt{\left[\frac{x_{0}^{2}(m+1)^{2}}{4}-j-1\right\rfloor}\right)\right),
$$

$$
\begin{aligned}
I^{*}= & \frac{\cos \left(\left(\pi / x_{0}\right) \sqrt{\left\lfloor\left(x_{0}^{2}(m+1)^{2} / 4\right)+j+1\right\rfloor}\right)}{\sqrt{\left\lfloor\left(x_{0}^{2}(m+1)^{2} / 4\right)+j+1\right\rfloor}} \\
& -\frac{4 x_{0}}{\pi} \sin \left(\frac{\pi}{2 x_{0}}\left(\sqrt{\left\lfloor\frac{x_{0}^{2}(m+1)^{2}}{4}+j+1\right\rfloor}-\sqrt{\left\lfloor\frac{x_{0}^{2}(m+1)^{2}}{4}+j\right\rfloor}\right)\right) \\
& * \cos \left(\frac{\pi}{2 x_{0}}\left(\sqrt{\left\lfloor\frac{x_{0}^{2}(m+1)^{2}}{4}+j+1\right\rfloor}+\sqrt{\left\lfloor\frac{x_{0}^{2}(m+1)^{2}}{4}+j\right\rfloor}\right)\right), \\
I^{* *}= & \frac{\cos \left(\left(\pi / x_{0}\right) \sqrt{\left\lfloor\left(x_{0}^{2}(m+1)^{2} / 4\right)-j\right\rfloor}\right)}{\sqrt{\left\lfloor\left(x_{0}^{2}(m+1)^{2} / 4\right)-j\right\rfloor}} \\
& -\frac{4 x_{0}}{\pi} \sin \left(\frac{\pi}{2 x_{0}}\left(\sqrt{\left\lfloor\frac{x_{0}^{2}(m+1)^{2}}{4}-j\right\rfloor}-\sqrt{\left.\mid \frac{x_{0}^{2}(m+1)^{2}}{4}-j-1\right\rfloor}\right)\right)
\end{aligned}
$$

where $m \in O d d_{+}=\{1,3,5,9, \cdots\}$.

Let $a, b \in R$ such that $0<a<b$. Then, for all $x_{0} \in[a, b]$ and for all $m>\max \left\{2 / x_{0}^{2}, 2 / x_{0}\right\}$,

$$
\sum_{j=0}^{\left\lfloor\left(x_{0}^{2} / 4\right)(2 m+1)-1\right\rfloor}\left|I^{*}+I^{* *}\right| \leq \frac{16 \pi^{2}}{x_{0}^{5} m^{2}}\left(2 x_{0}^{4}+x_{0}^{2}\right) .
$$

Proof. Let

$$
\begin{aligned}
& I_{1}^{*}= \frac{\cos \left(\left(\pi / x_{0}\right) \sqrt{\left\lfloor\left(x_{0}^{2}(m+1)^{2} / 4\right)+j+1\right\rfloor}\right)-\cos \left(\left(\pi / 2 x_{0}\right)\left(\sqrt{\left\lfloor\left(x_{0}^{2}(m+1)^{2} / 4\right)+j+1\right\rfloor}+\sqrt{\left\lfloor\left(x_{0}^{2}(m+1)^{2} / 4\right)+j\right\rfloor}\right)\right)}{\sqrt{\left\lfloor\left(x_{0}^{2}(m+1)^{2} / 4\right)+j+1\right\rfloor}}, \\
& I_{2}^{*}=\cos \left(\frac{\pi}{2 x_{0}}\left(\sqrt{\left\lfloor\frac{x_{0}^{2}(m+1)^{2}}{4}+j+1\right\rfloor}+\sqrt{\left.\left.\frac{\left\lfloor x_{0}^{2}(m+1)^{2}\right.}{4}+j\right\rfloor\right)}\right)\right. \\
& *\left\{\frac{1}{\sqrt{\left\lfloor\left(x_{0}^{2}(m+1)^{2} / 4\right)+j+1\right\rfloor}}-\frac{4 x_{0}}{\pi} \sin \left(\frac{\pi}{2 x_{0}}\left(\sqrt{\left.\frac{\left\lfloor x_{0}^{2}(m+1)^{2}\right.}{4}+j+1\right\rfloor}-\sqrt{\left.\left.\frac{\mid x_{0}^{2}(m+1)^{2}}{4}+j\right\rfloor\right)}\right\} .\right.\right.
\end{aligned}
$$

Note that for all $m>\max \left\{2 / x_{0}^{2}, 2 / x_{0}\right\}$,

$$
\begin{aligned}
& \frac{\pi}{2 x_{0}} \frac{1}{\sqrt{\left\lfloor\left(x_{0}^{2}(m+1)^{2} / 4\right)+j+1\right\rfloor}+\sqrt{\left\lfloor\left(x_{0}^{2}(m+1)^{2} / 4\right)+j\right\rfloor}}-\frac{1}{3 !}\left(\frac{\pi}{2 x_{0}} \frac{1}{\sqrt{\left\lfloor\left(x_{0}^{2}(m+1)^{2} / 4\right)+j+1\right\rfloor}+\sqrt{\left\lfloor\left(x_{0}^{2}(m+1)^{2} / 4\right)+j\right\rfloor}}\right)^{3} \\
& \leq \sin \left(\frac{\pi}{2 x_{0}}\left(\sqrt{\left\lfloor\frac{x_{0}^{2}(m+1)^{2}}{4}+j+1\right\rfloor}-\sqrt{\left\lfloor\frac{x_{0}^{2}(m+1)^{2}}{4}+j\right\rfloor}\right)\right) \leq \frac{\pi}{2 x_{0}} \frac{1}{\sqrt{\left\lfloor\left(x_{0}^{2}(m+1)^{2} / 4\right)+j+1\right\rfloor}+\sqrt{\left\lfloor\left(x_{0}^{2}(m+1)^{2} / 4\right)+j\right\rfloor}} .
\end{aligned}
$$

In view of (4), the following inequality is satisfied:

$$
\begin{aligned}
\left|I_{2}^{*}\right| & \leq \frac{\pi^{2}}{2 x_{0}^{5} 3 ! m^{3}}+\left|\frac{1}{\sqrt{\left\lfloor\left(x_{0}^{2}(m+1)^{2} / 4\right)+j+1\right\rfloor}}-\frac{2}{\sqrt{\left\lfloor\left(x_{0}^{2}(m+1)^{2} / 4\right)+j+1\right\rfloor}+\sqrt{\left\lfloor\left(x_{0}^{2}(m+1)^{2} / 4\right)+j\right\rfloor}}\right| \\
& \leq \frac{\pi^{2}}{2 x_{0}^{5} 3 ! m^{3}}+\frac{2}{m^{2} x_{0}^{4}}\left(\sqrt{\left.\mid \frac{x_{0}^{2}(m+1)^{2}}{4}+j\right\rfloor}-\sqrt{\left\lfloor\frac{x_{0}^{2}(m+1)^{2}}{4}+j+1\right\rfloor}\right) \leq \frac{8 \pi^{2}}{x_{0}^{5} m^{3}} .
\end{aligned}
$$


Let

$$
\begin{aligned}
& I_{1}^{* *}=\frac{\cos \left(\left(\pi / x_{0}\right) \sqrt{\left\lfloor\left(x_{0}^{2}(m+1)^{2} / 4\right)-j\right\rfloor}\right)-\cos \left(\left(\pi / 2 x_{0}\right)\left(\sqrt{\left\lfloor\left(x_{0}^{2}(m+1)^{2} / 4\right)-j\right\rfloor}+\sqrt{\left\lfloor\left(x_{0}^{2}(m+1)^{2} / 4\right)-j-1\right\rfloor}\right)\right)}{\sqrt{\left\lfloor\left(x_{0}^{2}(m+1)^{2} / 4\right)-j\right\rfloor}}, \\
& I_{2}^{* *}=\cos \left(\frac{\pi}{2 x_{0}}\left(\sqrt{\left\lfloor\frac{x_{0}^{2}(m+1)^{2}}{4}-j\right\rfloor}+\sqrt{\left\lfloor\frac{x_{0}^{2}(m+1)^{2}}{4}-j-1\right\rfloor}\right)\right) \\
& \text { * }\left\{\frac{1}{\sqrt{\left\lfloor\left(x_{0}^{2}(m+1)^{2} / 4\right)-j\right\rfloor}}-\frac{4 x_{0}}{\pi} \sin \left(\frac{\pi}{2 x_{0}}\left(\sqrt{\left\lfloor\frac{x_{0}^{2}(m+1)^{2}}{4}-j\right\rfloor}-\sqrt{\left\lfloor\frac{x_{0}^{2}(m+1)^{2}}{4}-j-1\right\rfloor}\right)\right)\right\}, \\
& I_{1}^{*}+I_{1}^{* *}=\frac{-2}{\sqrt{\left\lfloor\left(x_{0}^{2} / 4\right)(m+1)^{2}+j+1\right\rfloor}} \sin \left(\frac{\pi}{4 x_{0}}\left(\sqrt{\left\lfloor\frac{x_{0}^{2}}{4}(m+1)^{2}+j+1\right\rfloor}-\sqrt{\left\lfloor\frac{x_{0}^{2}}{4}(m+1)^{2}+j\right\rfloor}\right)\right) \\
& * \sin \left(\frac{\pi}{4 x_{0}}\left(\sqrt[3]{\left.\mid \frac{x_{0}^{2}}{4}(m+1)^{2}+j+1\right\rfloor}+\sqrt{\left[\frac{x_{0}^{2}}{4}(m+1)^{2}+j\right\rfloor}\right)\right) \\
& +\frac{-2}{\sqrt{\left\lfloor\left(x_{0}^{2} / 4\right)(m+1)^{2}-j\right\rfloor}} \sin \left(\frac{\pi}{4 x_{0}}\left(\sqrt{\left\lfloor\frac{x_{0}^{2}}{4}(m+1)^{2}-j\right\rfloor}-\sqrt{\left\lfloor\frac{x_{0}^{2}}{4}(m+1)^{2}-j-1\right\rfloor}\right)\right) \\
& * \sin \left(\frac{\pi}{4 x_{0}}\left(3 \sqrt{\left[\frac{x_{0}^{2}}{4}(m+1)^{2}-j\right\rfloor}+\sqrt{\left.\mid \frac{x_{0}^{2}}{4}(m+1)^{2}-j-1\right\rfloor}\right)\right) \\
& ==\frac{-2}{\sqrt{\left\lfloor\left(x_{0}^{2} / 4\right)(m+1)^{2}+j+1\right\rfloor}} \sin \left(\frac{\pi}{4 x_{0}}\left(\sqrt{\left\lfloor\frac{x_{0}^{2}}{4}(m+1)^{2}+j+1\right\rfloor}-\sqrt{\left\lfloor\frac{x_{0}^{2}}{4}(m+1)^{2}+j\right\rfloor}\right)\right) \\
& *\left\{\sin \left(\frac{\pi}{4 x_{0}}\left(\sqrt[3]{\left\lfloor\frac{x_{0}^{2}}{4}(m+1)^{2}+j+1\right\rfloor}+\sqrt{\left\lfloor\frac{x_{0}^{2}}{4}(m+1)^{2}+j\right\rfloor}\right)\right)+\sin \left(\frac{\pi}{4 x_{0}}\left(\sqrt[3]{\left\lfloor\frac{x_{0}^{2}}{4}(m+1)^{2}-j\right\rfloor}+\sqrt{\left\lfloor\frac{x_{0}^{2}}{4}(m+1)^{2}-j-1\right\rfloor}\right)\right)\right\} \\
& +\sin \left(\frac{\pi}{4 x_{0}}\left(\sqrt[3]{\left\lfloor\frac{x_{0}^{2}}{4}(m+1)^{2}-j\right\rfloor}+\sqrt{\left\lfloor\frac{x_{0}^{2}}{4}(m+1)^{2}-j-1\right\rfloor}\right)\right) *\left\{\frac { - 2 } { \sqrt { \lfloor ( x _ { 0 } ^ { 2 } / 4 ) ( m + 1 ) ^ { 2 } - j \rfloor } } \operatorname { s i n } \left(\frac { \pi } { 4 x _ { 0 } } \left(\sqrt{\left\lfloor\frac{x_{0}^{2}}{4}(m+1)^{2}-j\right\rfloor}\right.\right.\right. \\
& \left.\left.\left.-\sqrt{\left\lfloor\frac{x_{0}^{2}}{4}(m+1)^{2}-j-1\right\rfloor}\right)\right)+\frac{2}{\sqrt{\left\lfloor\left(x_{0}^{2} / 4\right)(m+1)^{2}+j+1\right\rfloor}} \sin \left(\frac{\pi}{4 x_{0}}\left(\sqrt{\left\lfloor\frac{x_{0}^{2}}{4}(m+1)^{2}+j+1\right\rfloor}-\sqrt{\left\lfloor\frac{x_{0}^{2}}{4}(m+1)^{2}+j\right\rfloor}\right)\right)\right\}=J_{1}+J_{2} \text {. }
\end{aligned}
$$

In analogy with (5), we have

$$
\left|I_{2}^{* *}\right| \leq \frac{8 \pi^{2}}{x_{0}^{5} m^{3}}
$$


If $D \in\{-3,-2,-1,0\}$, then

$$
\begin{aligned}
& \left|J_{1}\right| \leq \frac{\pi}{x_{0}^{3} m^{2}} \mid \sin \left(\frac{\pi}{4 x_{0}}\left(2 x_{0}(m+1)+3 \sqrt{\left\lfloor\frac{x_{0}^{2}}{4}(m+1)^{2}+j+1\right\rfloor}+\sqrt{\left\lfloor\frac{x_{0}^{2}}{4}(m+1)^{2}+j\right\rfloor}-2 x_{0}(m+1)\right)\right) \\
& +\sin \left(\frac{\pi}{4 x_{0}}\left(2 x_{0}(m+1)+3 \sqrt{\left\lfloor\frac{x_{0}^{2}}{4}(m+1)^{2}-j\right\rfloor}+\sqrt{\left\lfloor\frac{x_{0}^{2}}{4}(m+1)^{2}-j-1\right\rfloor}-2 x_{0}(m+1)\right)\right) \mid \\
& =\frac{\pi}{x_{0}^{3} m^{2}} \mid(-1)^{(m+1) / 2} \sin \left(\frac{\pi}{4 x_{0}}\left(3 \sqrt{\left\lfloor\frac{x_{0}^{2}}{4}(m+1)^{2}+j+1\right\rfloor}+\sqrt{\left.\mid \frac{x_{0}^{2}}{4}(m+1)^{2}+j\right\rfloor}-2 x_{0}(m+1)\right)\right) \\
& +(-1)^{(m+1) / 2} \sin \left(\frac{\pi}{4 x_{0}}\left(\sqrt[3]{\left\lfloor\frac{x_{0}^{2}}{4}(m+1)^{2}-j\right\rfloor}+\sqrt{\left\lfloor\frac{x_{0}^{2}}{4}(m+1)^{2}-j-1\right\rfloor}-2 x_{0}(m+1)\right)\right) \\
& =\frac{\pi}{x_{0}^{3} m^{2}} \mid \sin \left(\frac{\pi}{4 x_{0}}\left(\frac{9\left\lfloor\left(x_{0}^{2} / 4\right)(m+1)^{2}+j+1\right\rfloor-(9 / 4) x_{0}^{2}(m+1)^{2}}{3 \sqrt{\left\lfloor\left(x_{0}^{2} / 4\right)(m+1)^{2}+j+1\right\rfloor}+(3 / 2) x_{0}(m+1)}+\frac{\left\lfloor\left(x_{0}^{2} / 4\right)(m+1)^{2}+j\right\rfloor-(1 / 4) x_{0}^{2}(m+1)^{2}}{\sqrt{\left\lfloor\left(x_{0}^{2} / 4\right)(m+1)^{2}+j\right\rfloor}+(1 / 2) x_{0}(m+1)}\right)\right) \\
& +\sin \left(\frac{\pi}{4 x_{0}}\left(\frac{9\left\lfloor\left(x_{0}^{2} / 4\right)(m+1)^{2}-j\right\rfloor-(9 / 4) x_{0}^{2}(m+1)^{2}}{3 \sqrt{\left\lfloor\left(x_{0}^{2} / 4\right)(m+1)^{2}-j\right\rfloor}+(3 / 2) x_{0}(m+1)}+\frac{\left\lfloor\left(x_{0}^{2} / 4\right)(m+1)^{2}-j-1\right\rfloor-(1 / 4) x_{0}^{2}(m+1)^{2}}{\sqrt{\left\lfloor\left(x_{0}^{2} / 4\right)(m+1)^{2}-j-1\right\rfloor}+(1 / 2) x_{0}(m+1)}\right)\right) \\
& \leq \frac{\pi}{x_{0}^{3} m^{2}} \mid 2 \sin \left(\frac { \pi } { 8 x _ { 0 } } \left(\frac{3 j+3 D}{\sqrt{\left\lfloor\left(x_{0}^{2} / 4\right)(m+1)^{2}+j+1\right\rfloor}+(1 / 2) x_{0}(m+1)}+\frac{j+D}{\sqrt{\left\lfloor\left(x_{0}^{2} / 4\right)(m+1)^{2}+j\right\rfloor}+(1 / 2) x_{0}(m+1)}\right.\right. \\
& \left.\left.+\frac{-3 j+3 D}{\sqrt{\left\lfloor\left(x_{0}^{2} / 4\right)(m+1)^{2}-j\right\rfloor}+(1 / 2) x_{0}(m+1)}+\frac{-j+D}{\sqrt{\left\lfloor\left(x_{0}^{2} / 4\right)(m+1)^{2}-j-1\right\rfloor}+(1 / 2) x_{0}(m+1)}\right)\right) \mid \\
& \leq \frac{\pi^{2}}{4 x_{0}^{4} m^{2}}\left\{\left|3 j \frac{\sqrt{\left\lfloor\left(x_{0}^{2} / 4\right)(m+1)^{2}-j\right\rfloor}-\sqrt{\left\lfloor\left(x_{0}^{2} / 4\right)(m+1)^{2}+j+1\right\rfloor}}{\left(\sqrt{\left\lfloor\left(x_{0}^{2} / 4\right)(m+1)^{2}-j\right\rfloor}+(1 / 2) x_{0}(m+1)\right)\left(\sqrt{\left\lfloor\left(x_{0}^{2} / 4\right)(m+1)^{2}+j+1\right\rfloor}+(1 / 2) x_{0}(m+1)\right)}\right|\right. \\
& \left.+\left|j \frac{\sqrt{\left\lfloor\left(x_{0}^{2} / 4\right)(m+1)^{2}-j-1\right\rfloor}-\sqrt{\left\lfloor\left(x_{0}^{2} / 4\right)(m+1)^{2}+j\right\rfloor}}{\left(\sqrt{\left\lfloor\left(x_{0}^{2} / 4\right)(m+1)^{2}-j-1\right\rfloor}+(1 / 2) x_{0}(m+1)\right)\left(\sqrt{\left\lfloor\left(x_{0}^{2} / 4\right)(m+1)^{2}+j\right\rfloor}+(1 / 2) x_{0}(m+1)\right)}\right|\right\}+\frac{2 \pi^{2}}{x_{0}^{5} m^{3}} \leq \frac{16 \pi^{2}}{x_{0}^{7} m^{5}} j^{2}+\frac{2 \pi^{2}}{x_{0}^{5} m^{3}},
\end{aligned}
$$

$$
\begin{aligned}
& \left|J_{2}\right| \leq \mid \frac{\sin \left(\left(\pi / 4 x_{0}\right)\left(\sqrt{\left\lfloor\left(x_{0}^{2} / 4\right)(m+1)^{2}+j+1\right\rfloor}-\sqrt{\left\lfloor\left(x_{0}^{2} / 4\right)(m+1)^{2}+j\right\rfloor}\right)\right)}{\sqrt{\left\lfloor\left(x_{0}^{2} / 4\right)(m+1)^{2}-j\right\rfloor}} \\
& -2 \frac{\sin \left(\left(\pi / 4 x_{0}\right)\left(\sqrt{\left\lfloor\left(x_{0}^{2} / 4\right)(m+1)^{2}-j\right\rfloor}-\sqrt{\left\lfloor\left(x_{0}^{2} / 4\right)(m+1)^{2}-j-1\right\rfloor}\right)\right)}{\sqrt{\left\lfloor\left(x_{0}^{2} / 4\right)(m+1)^{2}-j\right\rfloor}} \\
& +\left|2 \sin \left(\frac{\pi}{4 x_{0}}\left(\sqrt{\left\lfloor\frac{x_{0}^{2}}{4}(m+1)^{2}+j+1\right\rfloor}-\sqrt{\left\lfloor\frac{x_{0}^{2}}{4}(m+1)^{2}+j\right\rfloor}\right)\right)\left\{\frac{1}{\sqrt{\left\lfloor\left(x_{0}^{2} / 4\right)(m+1)^{2}+j+1\right\rfloor}}-\frac{1}{\sqrt{\left\lfloor\left(x_{0}^{2} / 4\right)(m+1)^{2}-j\right\rfloor}}\right\}\right| \\
& \leq \frac{4}{x_{0} m}\left|2 \sin \left(\frac{\pi}{8 x_{0}}\left(\sqrt{\left\lfloor\frac{x_{0}^{2}}{4}(m+1)^{2}+j+1\right\rfloor}-\sqrt{\left\lfloor\frac{x_{0}^{2}}{4}(m+1)^{2}+j\right\rfloor}-\sqrt{\left.\mid \frac{x_{0}^{2}}{4}(m+1)^{2}-j\right\rfloor}+\sqrt{\left.\mid \frac{x_{0}^{2}}{4}(m+1)^{2}-j-1\right\rfloor}\right)\right)\right|+\frac{4 \pi(j+1)}{x_{0}^{5} m^{4}} \\
& \leq \frac{\pi}{x_{0}^{2} m}\left|\frac{1}{\sqrt{\left\lfloor\left(x_{0}^{2} / 4\right)(m+1)^{2}+j+1\right\rfloor}+\sqrt{\left\lfloor\left(x_{0}^{2} / 4\right)(m+1)^{2}+j\right\rfloor}}-\frac{1}{\sqrt{\left\lfloor\left(x_{0}^{2} / 4\right)(m+1)^{2}-j\right\rfloor}+\sqrt{\left\lfloor\left(x_{0}^{2} / 4\right)(m+1)^{2}-j-1\right\rfloor}}\right|+\frac{4 \pi(j+1)}{x_{0}^{5} m^{4}}
\end{aligned}
$$

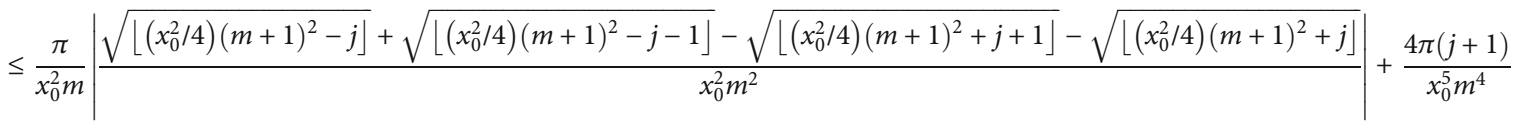

$$
\begin{aligned}
& \leq \frac{\pi}{x_{0}^{4} m^{3}}\left(\frac{2 j}{x_{0} m}+\frac{2 j+2}{x_{0} m}\right)+\frac{4 \pi(j+1)}{x_{0}^{5} m^{4}} \leq \frac{6 \pi(j+1)}{x_{0}^{5} m^{4}} .
\end{aligned}
$$


In view of (5) and (8)-(11), the following inequality is satisfied:

$$
\left|I^{*}+I^{* *}\right| \leq \frac{16 \pi^{2}}{x_{0}^{5} m^{2}}\left(\frac{1}{m}+\frac{j}{m^{2}}+\frac{j^{2}}{x_{0}^{2} m^{3}}\right) .
$$
have

Then, for all $x_{0} \in[a, b]$ and $m>\max \left\{2 / x_{0}^{2}, 2 / x_{0}\right\}$, we

$$
\sum_{j=0}^{\left\lfloor\left(x_{0}^{2} / 4\right)(2 m+1)-1\right\rfloor}\left|I^{*}+I^{* *}\right| \leq \frac{16 \pi^{2}}{x_{0}^{5} m^{2}}\left(2 x_{0}^{4}+x_{0}^{2}\right) .
$$

In the recent paper [4], it was proved, among others, that if $\left\{c_{n}\right\}$ is a nonincreasing sequence and $\sum_{n=1} c_{n} \cos (\sqrt{n} x)$ is convergent at any point $x \neq 0$, then $\lim _{n \rightarrow \infty} \sqrt{n} c_{n}=0$. We show that if $\left\{\sqrt{n} c_{n}\right\}$ is nonincreasing and $\lim _{n \rightarrow \infty} \sqrt{n} c_{n}=$ 0 , then $\sum_{n=1} c_{n} \cos (x \sqrt{n})$ converges uniformly on $[c, d]$, where $c>0$.

Theorem 6. Let $c, d \in R$ such that $0<c<d$. If $\left\{n a_{n}\right\}_{n=1}^{\infty}$ is nonincreasing and $\lim _{n \rightarrow \infty} n a_{n}=0$, then the series

$$
f(x)=\sum_{n=1}^{\infty} a_{n} \sin (x \sqrt{n}),
$$

can be differentiated term-by-term on $[c, d]($ or $[-d,-c])$. The series

$$
f^{\prime}(x)=\sum_{n=1}^{\infty} a_{n} \sqrt{n} \cos (x \sqrt{n})
$$

converges uniformly on $[c, d]$ (or $[-d,-c])$.

Proof. We can find $a, b \in R$ such that $0<a<b, c=\pi / b$, and $d=\pi / a$. If $\left\{a_{n}\right\}_{n=1}^{\infty}$ is a nonnegative monotone sequence and $\lim _{n \rightarrow \infty} n a_{n}=0$, then the series (14) converges uniformly [9]. We show that if, in addition, $\left\{n a_{n}\right\}_{n=1}^{\infty}$ is nonin- creasing, then (for $x=\pi / x_{0}$ ) the series $\sum_{n=1}^{\infty} a_{n} \sqrt{n} \cos \left(\pi \sqrt{n} / x_{0}\right)$ converges uniformly on $[a, b]$. Let $m \in \operatorname{Odd}+, b_{n}=\cos \left(\pi \sqrt{n} / x_{0}\right) / \sqrt{n}, B_{k}=\sum_{n=1}^{k} b_{n}$, and $M_{m}=$ $\left\{\left\lfloor\left(x_{0}^{2} / 4\right) m^{2}\right\rfloor+1,\left\lfloor\left(x_{0}^{2} / 4\right) m^{2}\right\rfloor+2, \cdots,\left\lfloor\left(x_{0}^{2} / 4\right)(m+2)^{2}\right\rfloor\right\}$. We denote by $m_{0}$ the minimal odd number for which there holds the following: $4 \mid m_{0}-1$ and $m_{0} \geq \max \left\{2 / x_{0}^{2}, 2 / x_{0}\right\}$. Then,

$$
\exists H^{\prime} \forall x_{0} \in[a, b] \sum_{n=1}^{\left\lfloor x_{0}^{2} m_{0}^{2} / 4\right\rfloor} b_{n}<H^{\prime} .
$$

Let

$$
\left.B_{k}^{\prime \prime}=\sum_{n=\left\lfloor\left(x_{0}^{2} / 4\right)\right.}^{k} b_{n}^{2}+1\right\rfloor
$$

Note that for all $m$ such that $4 \mid m-1$ and for all $k \in M_{m}$, the following conditions are fulfilled: $b_{k} \leq 0$ and

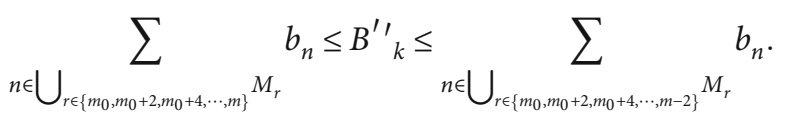

On the other hand, for all $m$ such that $4 \mid m-3$ and for all $k \in M_{m}$, we have $b_{k} \geq 0$ and

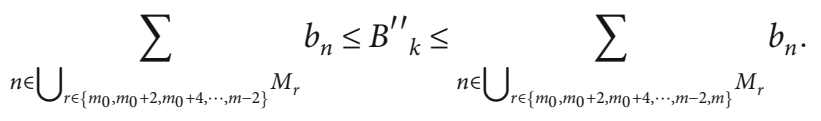

We show that the sequence

$$
\sum_{n \in \bigcup_{r \in\left\{m_{0}, m_{0}+2, m_{0}+4, \cdots, m-2, m\right\}} M_{r}} b_{n}
$$

is bounded for $4 \mid m-1$ and $4 \mid m-3$.

$$
\begin{aligned}
& \sum_{n \in \bigcup_{r \in\left\{m_{0}, m_{0}+2,2, m_{0}+4, \ldots, m-2, m\right\rfloor} M_{r}} b_{n}=\sum_{r \in \mathrm{Odd}, r=m_{0}, \cdots, m} \sum_{j=1}^{\left\lfloor\left(x_{0}^{2} / 4\right)(r+2)^{2}\right\rfloor-\left\lfloor\left(x_{0}^{2} / 4\right) r^{2}\right\rfloor} \frac{\cos \left(\left(\pi / x_{0}\right) \sqrt{\left.\left\lfloor\left(x_{0}^{2} / 4\right) r^{2}+j\right\rfloor\right)}\right.}{\sqrt{\left\lfloor\left(x_{0}^{2} / 4\right) r^{2}+j\right\rfloor}} \\
& =\sum_{r \in \mathrm{Odd}_{+}, r=m_{0}, \cdots, m}\left\{\sum_{j=0}^{\left\lfloor\left(x_{0}^{2} / 4\right)(2 r+1)-1\right\rfloor}\left(\frac{\cos \left(\left(\pi / x_{0}\right) \sqrt{\left\lfloor\left(x_{0}^{2} / 4\right)(r+1)^{2}+j+1\right\rfloor}\right)}{\sqrt{\left\lfloor\left(x_{0}^{2} / 4\right)(r+1)^{2}+j+1\right\rfloor}}+\frac{\cos \left(\left(\pi / x_{0}\right) \sqrt{\left\lfloor\left(x_{0}^{2} / 4\right)(r+1)^{2}-j\right\rfloor}\right)}{\sqrt{\left\lfloor\left(x_{0}^{2} / 4\right)(r+1)^{2}-j\right\rfloor}}\right)\right. \\
& \left.+\sum_{j=1}^{\left\lfloor\left(x_{0}^{2} / 4\right)(r+1)^{2}\right\rfloor-\left\lfloor\left(x_{0}^{2} / 4\right)(2 r+1)\right\rfloor\left\lfloor\left\lfloor\left(x_{0}^{2} / 4\right)^{2}\right\rfloor\right.} \frac{\cos \left(\left(\pi / x_{0}\right) \sqrt{\left\lfloor\left(x_{0}^{2} / 4\right) r^{2}+j\right\rfloor}\right)}{\sqrt{\left\lfloor\left(x_{0}^{2} / 4\right) r^{2}+j\right\rfloor}}+\sum_{j=\left\lfloor\left(x_{0}^{2} / 4\right)(r+1)^{2}\right\rfloor+\left\lfloor\left(x_{0}^{2} / 4\right)(2 r+1)\right\rfloor\left\lfloor\left(x_{0}^{2} / 4\right) r^{2}\right\rfloor}^{\left\lfloor\left(x_{0}^{2} / 4\right)(r+2)^{2}\right\rfloor\left\lfloor\left(x_{0}^{2} / 4\right) r^{2}\right\rfloor} \frac{\cos \left(\left(\pi / x_{0}\right) \sqrt{\left\lfloor\left(x_{0}^{2} / 4\right) r^{2}+j\right\rfloor}\right)}{\sqrt{\left\lfloor\left(x_{0}^{2} / 4\right) r^{2}+j\right\rfloor}}\right\} \\
& =\sum_{r \in \mathrm{Odd}_{+}, r=m_{0}, \cdots, m}\left\{S_{1}+S_{2}+S_{3}\right\} \text {. }
\end{aligned}
$$


Note that $\left(x_{0}^{2} / 2\right)-1 \leq\left\lfloor\left(x_{0}^{2} / 4\right)(r+2)^{2}\right\rfloor-\left\lfloor\left(x_{0}^{2} / 4\right)(r+1)^{2}\right.$ \rfloor$-\left\lfloor\left(x_{0}^{2} / 4\right)(2 r+1)\right\rfloor \leq\left(x_{0}^{2} / 2\right)+2$. Hence,

$$
\begin{aligned}
\left|S_{3}\right| \leq & \frac{\left(2+\left(x_{0}^{2} / 2\right)\right)}{x_{0} r}\left|\cos \left(\frac{\pi}{x_{0}} \sqrt{\left.\mid \frac{x_{0}^{2}}{4}(r+1)^{2}\right\rfloor+\left\lfloor\frac{x_{0}^{2}}{4}(2 r+1)\right\rfloor}\right)\right| \\
\leq & 2 \frac{4+x_{0}}{x_{0} r} \mid \cos \left(\frac { \pi } { x _ { 0 } } \left(\frac{x_{0}}{2}(r+2)+\sqrt{\left.\mid \frac{x_{0}^{2}}{4}(r+1)^{2}\right\rfloor+\left\lfloor\frac{x_{0}^{2}}{4}(2 r+1)\right\rfloor}\right.\right. \\
& \left.\left.-\frac{x_{0}}{2}(r+2)\right)\right) \mid \leq 2 \frac{\pi\left(x_{0}^{2} / 2+2\right)\left(4+x_{0}\right)}{x_{0}^{3} r^{2}} .
\end{aligned}
$$

\section{Moreover,}

$$
\left|S_{2}\right| \leq \frac{2}{x_{0} r / 2}\left|\cos \frac{\pi}{x_{0}} \sqrt{\left|\frac{x_{0}^{2}}{4} r^{2}+2\right|}\right| \leq \frac{4 \pi}{x_{0}^{3} r^{2}} .
$$

\section{Furthermore,}

$$
\begin{aligned}
S_{1}= & \sum_{j=0}^{\left\lfloor\left(x_{0}^{2} / 4\right)(2 r+1)-1\right\rfloor}\left\{\frac{4 x_{0}}{\pi} \sin \left(\frac{\pi}{2 x_{0}}\left(\sqrt{\left\lfloor\frac{x_{0}}{4}(r+1)^{2}+j+1\right\rfloor}-\sqrt{\left\lfloor\frac{x_{0}}{4}(r+1)^{2}+j\right\rfloor}\right)\right) * \cos \left(\frac{\pi}{2 x_{0}}\left(\sqrt{\left\lfloor\frac{x_{0}}{4}(r+1)^{2}+j+1\right\rfloor}+\sqrt{\left\lfloor\frac{x_{0}}{4}(r+1)^{2}+j\right\rfloor}\right)\right)\right. \\
& \left.+\frac{4 x_{0}}{\pi} \sin \left(\frac{\pi}{2 x_{0}}\left(\sqrt{\left\lfloor\frac{x_{0}}{4}(r+1)^{2}-j\right\rfloor}-\sqrt{\left\lfloor\frac{x_{0}}{4}(r+1)^{2}-j-1\right\rfloor}\right)\right) * \cos \left(\frac{\pi}{2 x_{0}}\left(\sqrt{\left\lfloor\frac{x_{0}}{4}(r+1)^{2}-j\right\rfloor}+\sqrt{\left\lfloor\frac{x_{0}}{4}(r+1)^{2}-j-1\right\rfloor}\right)\right)+I^{*}+I^{* *}\right\} \\
= & S_{1}^{\prime}+\sum_{j=0}^{\left\lfloor\left(x_{0}^{2 / 4}\right)(2 r+1)-1\right\rfloor}\left(I^{*}+I^{* *}\right),
\end{aligned}
$$

$$
\begin{aligned}
& S_{1}^{\prime}=\sum_{j=0}^{\left\lfloor\left(x_{0}^{2} / 4\right)(2 r+1)-1\right\rfloor}\left\{\frac{2 x_{0}}{\pi}\left[\sin \left(\frac{\pi}{x_{0}} \sqrt{\left\lfloor\frac{x_{0}^{2}}{4}(r+1)^{2}+j+1\right\rfloor}\right)-\sin \left(\frac{\pi}{x_{0}} \sqrt{\left\lfloor\frac{x_{0}^{2}}{4}(r+1)^{2}+j\right\rfloor}\right)\right]+\frac{2 x_{0}}{\pi}\left[\sin \left(\frac{\pi}{x_{0}} \sqrt{\left\lfloor\frac{x_{0}^{2}}{4}(r+1)^{2}-j\right\rfloor}\right)\right.\right. \\
& \left.\left.-\sin \left(\frac{\pi}{x_{0}} \sqrt{\left[\frac{x_{0}^{2}}{4}(r+1)^{2}-j-1 \mid\right.}\right)\right]\right\}=\frac{2 x_{0}}{\pi}\left[\sin \left(\frac{\pi}{x_{0}} \sqrt{\left.\mid \frac{x_{0}^{2}}{4}(r+1)^{2}\right\rfloor+\left[\frac{x_{0}^{2}}{4}(2 r+1)\right\rfloor}\right)-\sin \left(\frac{\pi}{x_{0}} \sqrt{\left[\frac{x_{0}^{2}}{4}(r+1)^{2}\right\rfloor}\right)\right. \\
& \left.-\sin \left(\frac{\pi}{x_{0}} \sqrt{\left[\frac{x_{0}^{2}}{4}(r+1)^{2}\right\rfloor-\left\lfloor\frac{x_{0}^{2}}{4}(2 r+1)\right\rfloor}\right)+\sin \left(\frac{\pi}{x_{0}} \sqrt{\left\lfloor\frac{x_{0}^{2}}{4}(r+1)^{2}\right\rfloor}\right)\right] \\
& =\frac{2 x_{0}}{\pi}\left[(-1)^{(r+1) / 2} \cos \left(\frac{\pi}{x_{0}} \sqrt{\left[\frac{x_{0}^{2}}{4}(r+1)^{2}\right\rfloor+\left\lfloor\frac{x_{0}^{2}}{4}(2 r+1)\right\rfloor}-\frac{(r+2) \pi}{2}\right)+(-1)^{(r+1) / 2} \cos \left(\frac{\pi}{x_{0}} \sqrt{\left\lfloor\frac{x_{0}^{2}}{4}(r+1)^{2}\right\rfloor-\left\lfloor\frac{x_{0}^{2}}{4}(2 r+1)\right\rfloor}-\frac{r \pi}{2}\right)\right] \\
& =\frac{2 x_{0}}{\pi}(-1)^{(r+1) / 2}\left[1-\left(1-\cos \left(\frac{\pi}{x_{0}} \sqrt{\left\lfloor\frac{x_{0}^{2}}{4}(r+1)^{2}\right\rfloor+\left\lfloor\frac{x_{0}^{2}}{4}(2 r+1)\right\rfloor}-\frac{(r+2) \pi}{2}\right)\right)+1-\left(1-\cos \left(\frac{\pi}{x_{0}} \sqrt{\left[\frac{x_{0}^{2}}{4}(r+1)^{2}\right\rfloor-\left\lfloor\frac{x_{0}^{2}}{4}(2 r+1)\right\rfloor}-\frac{r \pi}{2}\right)\right)\right] \\
& =\frac{4 x_{0}}{\pi}(-1)^{(r+1) / 2}-\frac{2 x_{0}}{\pi}(-1)^{(r+1) / 2}\left[\frac{1-\cos ^{2}\left(\left(\pi / x_{0}\right) \sqrt{\left\lfloor\left(x_{0}^{2} / 4\right)(r+1)^{2}\right\rfloor+\left\lfloor\left(x_{0}^{2} / 4\right)(2 r+1)\right\rfloor}-((r+2) \pi / 2)\right)}{1+\cos \left(\left(\pi / x_{0}\right) \sqrt{\left\lfloor\left(x_{0}^{2} / 4\right)(r+1)^{2}\right\rfloor+\left\lfloor\left(x_{0}^{2} / 4\right)(2 r+1)\right\rfloor}-((r+2) \pi / 2)\right)}++\frac{1-\cos ^{2}\left(\left(\pi / x_{0}\right) \sqrt{\left\lfloor\left(x_{0}^{2} / 4\right)(r+1)^{2}\right\rfloor-\left\lfloor\left(x_{0}^{2} / 4\right)(2 r+1)\right\rfloor}-(r \pi / 2)\right)}{1+\cos \left(\left(\pi / x_{0}\right) \sqrt{\left\lfloor\left(x_{0}^{2} / 4\right)(r+1)^{2}\right\rfloor-\left\lfloor\left(x_{0}^{2} / 4\right)(2 r+1)\right\rfloor}-(r \pi / 2)\right)}\right] .
\end{aligned}
$$

Note that

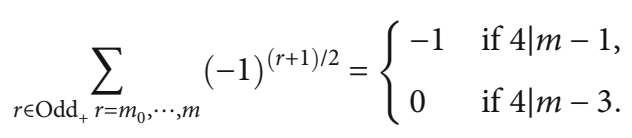


In view of Lemma 5 and (22)-(26), the following inequality is satisfied:

$$
\begin{aligned}
\left|\sum_{r \in \mathrm{Odd}_{+}, r=m_{0}, \cdots, m}\left(S_{1}+S_{2}+S_{3}\right)\right| \leq & \left|\sum_{r \in \mathrm{Odd}_{+}, r=m_{0}, \cdots, m} \frac{4 x_{0}}{\pi}(-1)^{(r+1) / 2}\right|+\sum_{r \in \mathrm{Odd}_{+}, r=m_{0}, \cdots, m}\left\{\frac { 2 x _ { 0 } } { \pi } \left[\operatorname { s i n } ^ { 2 } \left(\frac{\pi}{x_{0}} \sqrt{\left.\left.\left.\mid \frac{x_{0}^{2}}{4}(r+1)^{2}\right\rfloor+\mid \frac{x_{0}^{2}}{4}(2 r+1)\right\rfloor-\frac{(r+2) \pi}{2}\right)}\right.\right.\right. \\
& +\sin ^{2}\left(\frac{\pi}{x_{0}} \sqrt{\left.\left.\mid \frac{x_{0}^{2}}{4}(r+1)^{2}\right\rfloor-\left\lfloor\frac{x_{0}^{2}}{4}(2 r+1) \mid-\frac{r \pi}{2}\right)\right]+\mid\left\lfloor\left(x_{0}^{2} / 4\right)(2 r+1)-1\right\rfloor} \sum_{j=0}\left(I^{*}+I^{* *}\right)|+| S_{2}|+| S_{3} \mid\right\} \\
\leq & \frac{4 x_{0}}{\pi}+\sum_{r \in \mathrm{Odd}_{+}, r=m_{0}, \cdots, m}\left\{\frac{16 \pi^{2}}{r^{2} x_{0}^{5}}\left(2 x_{0}^{4}+x_{0}^{2}\right)+\frac{16 x_{0}}{r^{2} \pi}\left(\frac{\pi^{2}}{4}+\frac{\pi^{2}}{x_{0}}\right)^{2}+\frac{4 \pi}{r^{2} x_{0}^{3}}+\frac{2 \pi\left(\left(x_{0}^{2} / 2\right)+2\right)\left(4+x_{0}\right)}{r^{2} x_{0}^{3}}\right\}<H,
\end{aligned}
$$

for all $x_{0} \in[a, b]$. Using (17)-(19), (21), and (27), we obtain

$$
\exists H^{\prime \prime} \forall x_{0} \in[a, b] B_{k}^{\prime \prime}<H^{\prime \prime} .
$$

In view of (16) and (28), we get

$$
\exists G \forall x_{0} \in[a, b] B_{k}<G .
$$

We know that $\left\{n a_{n}\right\}$ is nonincreasing and $\lim _{n \rightarrow \infty} n a_{n}$ $=0$ and (29) holds. Hence, the series $\sum n a_{n}\left(\cos \left(\pi \sqrt{n} / x_{0}\right) /\right.$ $\sqrt{n})$ converges uniformly. This follows from Dirichlet's test.

Remark 7. The conditions $\left(\left\{n a_{n}\right\}\right.$ is nonincreasing, and $\left.\lim _{n \rightarrow \infty} n a_{n}=0\right)$ are not sufficient for the differentiability of (14) at the point $x=0$.

Example 1. Let $f(x)=\sum_{n=4}(\sin (x \sqrt{n}) / n \ln n)$. It converges uniformly on $[0, \pi][5]$. We show that $f$ is not differentiable at $x=0$. Let $f(\Delta x)=\sum_{n=4}(1 / n \ln n) \sin (\pi \sqrt{n} / 2 \alpha)$ for $\Delta x=\pi / 2$ $\alpha$ and $\alpha \in \operatorname{Odd}_{+}: 4 \mid \alpha-1, \alpha \geq 5$. Then, by Lagrange's theorem,

$$
\begin{aligned}
& \sum_{n=4}^{\alpha^{2}} \frac{1}{n \ln n} \sin \frac{\pi \sqrt{n}}{2 \alpha} \geq \frac{1}{\alpha \ln \alpha^{2}} \sum_{n=4}^{\alpha^{2}} \frac{1}{\sqrt{n}} \sin \frac{\pi \sqrt{n}}{2 \alpha} \\
& \geq \frac{1}{\alpha \ln \alpha^{2}} \int_{3}^{\alpha^{2}} \frac{1}{\sqrt{n}} \sin \frac{\pi \sqrt{n}}{2 \alpha} d n \quad(30) \\
& =\frac{4}{\pi \ln \alpha^{2}} \cos \frac{\pi \sqrt{3}}{2 \alpha}, \\
& \sum_{n=\alpha^{2}+1}^{(2 \alpha)^{2}} \frac{1}{n \ln n} \sin \frac{\pi \sqrt{n}}{2 \alpha} \geq \frac{1}{2 \alpha \ln (2 \alpha)^{2}} \sum_{n=\alpha^{2}+1}^{(2 \alpha)^{2}} \frac{1}{\sqrt{n}} \sin \frac{\pi \sqrt{n}}{2 \alpha} \\
& \geq \frac{1}{2 \alpha \ln (2 \alpha)^{2}} \int_{\alpha^{2}+1}^{(2 \alpha)^{2}+1} \frac{1}{\sqrt{n} \sin \frac{\pi \sqrt{n}}{2 \alpha}} d n \\
& =\frac{-4}{2 \pi \ln (2 \alpha)^{2}}\left[\frac{\pi \sqrt{(2 \alpha)^{2}+1}}{2 \alpha} \cos \frac{\pi \sqrt{(\alpha)^{2}+1}}{2 \alpha}\right]
\end{aligned}
$$

The function $\sin x / x$ has a relative minimum at the point $x_{1} \in(\pi, 3 \pi / 2)$. Then,

$$
\begin{aligned}
& \sum_{n=(2 \alpha)^{2}+1}^{\left\lfloor\left(x_{1} 2 \alpha / \pi\right)^{2}\right\rfloor} \frac{1}{n \ln n} \sin \frac{\pi \sqrt{n}}{2 \alpha} \\
& \quad \geq \frac{-4}{2 \pi \ln (2 \alpha)^{2}}\left[\cos \frac{\pi \sqrt{\left\lfloor\left(x_{1} 2 \alpha / \pi\right)^{2}+1\right\rfloor}}{2 \alpha}-\cos \frac{\pi \sqrt{(2 \alpha)^{2}+1}}{2 \alpha}\right],
\end{aligned}
$$

$$
\begin{aligned}
n=\left\lfloor\left(x_{1} 2 \alpha / \pi\right)^{2}\right\rfloor+1 & \frac{1}{n \ln n} \sin \frac{\pi \sqrt{n}}{2 \alpha} \\
= & \sum_{n=\left\lfloor\left(x_{1} 2 \alpha / \pi\right)^{2}\right\rfloor+2}^{(3 \alpha)^{2}} \frac{1}{n \ln n} \sin \frac{\pi \sqrt{n}}{2 \alpha} \\
& +\frac{\sin \left(\pi \sqrt{\left.\left\lfloor\left(x_{1} 2 \alpha / \pi\right)^{2}+1\right\rfloor / 2 \alpha\right)}\right.}{\geq} \\
& \frac{\left.4\left(x_{1} 2 \alpha / \pi\right)^{2}+1\right\rfloor \ln \left\lfloor\left(x_{1} 2 \alpha / \pi\right)^{2}+1\right\rfloor}{2 \pi \ln (2 \alpha)^{2}} \cos \frac{\pi \sqrt{\left\lfloor\left(x_{1} 2 \alpha / \pi\right)^{2}+1\right\rfloor}}{2 \alpha}-\frac{1}{\alpha^{2} \ln \alpha^{2}} \\
& \sum_{n=(3 \alpha)^{2}+1}^{(4 \alpha)^{2}} \frac{1}{\ln n} \sin \frac{\pi \sqrt{n}}{2 \alpha} \geq \frac{-4}{3 \pi \ln (3 \alpha)^{2}}
\end{aligned}
$$

In view of (30)-(34), we obtain

$$
\sum_{n=4}^{(4 \alpha)^{2}} \frac{1}{n \ln n} \sin \frac{\pi \sqrt{n}}{2 \alpha} \geq \frac{1}{3 \pi \ln \alpha}-\frac{1}{\alpha^{2} \ln \alpha^{2}}
$$

If $x_{r}^{\prime}$ and $x_{r}{ }^{\prime \prime}$ are the relative minimum and maximum (respectively) points of $\sin x / x$, such that 


$$
\begin{aligned}
& x_{r}{ }^{\prime \prime} \in\left(2 \pi+2 r \pi, \frac{5 \pi}{2}+2 r \pi\right), \\
& x_{r}^{\prime} \in\left(3 \pi+2 r \pi, \frac{7 \pi}{2}+2 r \pi\right),
\end{aligned}
$$

for $r=0,1,2,3, \cdots$, then

$$
\begin{aligned}
& \sum_{n=\alpha^{2}+1}^{\infty} \frac{\sin (\pi \sqrt{n} / 2 \alpha)}{n \ln n}=\sum_{r=0}\left\{\sum_{n=(4(r+1) \alpha)^{2}+1}^{\left\lfloor\left(x_{r}^{\prime \prime} 2 \alpha / \pi\right)^{2}\right\rfloor} \frac{\sin (\pi \sqrt{n} / 2 \alpha)}{n \ln n}+\sum_{n=\left\lfloor\left(x_{r}^{\prime \prime} 2 \alpha / \pi\right)^{2}+1\right\rfloor}^{((4 r+5) \alpha)^{2}} \frac{\sin (\pi \sqrt{n} / 2 \alpha)}{n \ln n}+\sum_{n=((4 r+5) \alpha)^{2}+1}^{((4 r+6) \alpha)^{2}} \frac{\sin (\pi \sqrt{n} / 2 \alpha)}{n \ln n}\right. \\
& \left.+\sum_{n=(4 r+6) \alpha)^{2}+1}^{\left\lfloor\left(x_{r}^{\prime} 2 \alpha / \pi\right)^{2}\right\rfloor} \frac{\sin (\pi \sqrt{n} / 2 \alpha)}{n \ln n}+\sum_{n=\left\lfloor\left(x_{r}^{\prime} 2 \alpha / \pi\right)^{2}+1\right.}^{((4 r+7) \alpha)^{2}} \frac{\sin (\pi \sqrt{n} / 2 \alpha)}{n \ln n}+\sum_{n=((4 r+7) \alpha)^{2}+1}^{((4 r+8) \alpha)^{2}} \frac{\sin (\pi \sqrt{n} / 2 \alpha)}{n \ln n}\right\} \\
& \geq \sum_{r=0}\left\{\frac{1}{(4 r+5) \alpha \ln ((4 r+5) \alpha)^{2}}\left(\sum_{n=(4(r+1) \alpha)^{2}+1}^{\left\lfloor\left(x_{r}^{\prime \prime} 2 \alpha / \pi\right)^{2}\right\rfloor} \frac{\sin (\pi \sqrt{n} / 2 \alpha)}{\sqrt{n}}++\sum_{n=\left\lfloor\left(x_{r}^{\prime \prime} \alpha \alpha / \pi\right)^{2}+1\right\rfloor}^{((4 r+5) \alpha)^{2}} \frac{\sin (\pi \sqrt{n} / 2 \alpha)}{\sqrt{n}}\right)\right. \\
& +\frac{1}{(4 r+6) \alpha \ln ((4 r+6) \alpha)^{2}}\left(\sum_{n=((4 r+5) \alpha)^{2}+1}^{((4 r+6) \alpha)^{2}} \frac{\sin (\pi \sqrt{n} / 2 \alpha)}{\sqrt{n}}+\sum_{n=(4 r+6) \alpha)^{2}+1}^{\left\lfloor\left(x_{r}^{\prime} 2 \alpha / \pi\right)^{2}\right\rfloor} \frac{\sin (\pi \sqrt{n} / 2 \alpha)}{\sqrt{n}}+\sum_{n=\left\lfloor\left(x_{r}^{\prime} 2 \alpha / \pi\right)^{2}+1\right\rfloor}^{((4 r+7) \alpha)^{2}} \frac{\sin (\pi \sqrt{n} / 2 \alpha)}{\sqrt{n}}\right) \\
& \left.+\frac{1}{(4 r+7) \alpha \ln ((4 r+7) \alpha)^{2}} \sum_{n=((4 r+7) \alpha)^{2}+1}^{((4 r+8) \alpha)^{2}} \frac{\sin (\pi \sqrt{n} / 2 \alpha)}{\sqrt{n}}\right\} .
\end{aligned}
$$

In view of (36), we obtain

$$
\begin{aligned}
& \quad \sum_{n=(4(r+1) \alpha)^{2}+1}^{\left\lfloor\left(x_{r}^{\prime \prime} 2 \alpha / \pi\right)^{2}\right\rfloor} \frac{\sin (\pi \sqrt{n} / 2 \alpha)}{\sqrt{n}}+\sum_{n=\left\lfloor\left(x_{r}^{\prime \prime} 2 \alpha / \pi\right)^{2}+1\right\rfloor}^{((4 r+5) \alpha)^{2}} \frac{\sin (\pi \sqrt{n} / 2 \alpha)}{\sqrt{n}} \\
& \geq \int_{(4(r+1) \alpha)^{2}}^{\left\lfloor\left(x_{r}^{\prime \prime} 2 \alpha / \pi\right)^{2}\right\rfloor} \frac{\sin (\pi \sqrt{n} / 2 \alpha)}{\sqrt{n}} d n+\int_{\left\lfloor\left(x_{r}^{\prime \prime} 2 \alpha / \pi\right)^{2}+1\right\rfloor}^{((4 r+5) \alpha)^{2}-1} \frac{\sin (\pi \sqrt{n} / 2 \alpha)}{\sqrt{n}} d n \\
& =\int_{(4(r+1) \alpha)^{2}}^{((4 r+5) \alpha)^{2}} \frac{\sin (\pi \sqrt{n} / 2 \alpha)}{\sqrt{n}} d n-\int_{\left\lfloor\left(x_{r}^{\prime \prime} 2 \alpha / \pi\right)^{2}\right\rfloor}^{\left\lfloor\left(x_{r}^{\prime} \alpha /\right)^{2}\right\rfloor+1} \frac{\sin (\pi \sqrt{n} / 2 \alpha)}{\sqrt{n}} d n \\
& \quad-\int_{((4 r+5) \alpha)^{2}-1}^{((4 r+5) \alpha)^{2}} \frac{\sin (\pi \sqrt{n} / 2 \alpha)}{\sqrt{n}} d n \geq \frac{4 \alpha}{\pi}-\frac{2}{(r+1) \alpha},
\end{aligned}
$$

$$
\begin{aligned}
& \sum_{n=((4 r+5) \alpha)^{2}+1}^{((4 r+6) \alpha)^{2}} \frac{\sin (\pi \sqrt{n} / 2 \alpha)}{\sqrt{n}}+\sum_{n=(4 r+6) \alpha)^{2}+1}^{\left\lfloor\left(x_{r}^{\prime} 2 \alpha / \pi\right)^{2}\right\rfloor} \frac{\sin (\pi \sqrt{n} / 2 \alpha)}{\sqrt{n}} \\
& \quad+\sum_{n=\left\lfloor\left(x_{r}^{\prime} 2 \alpha / \pi\right)^{2}+1\right\rfloor} \frac{\sin (\pi \sqrt{n} / 2 \alpha)}{\sqrt{n}} \\
& \geq \int_{((4 r+5) \alpha)^{2}}^{((4 r+6) \alpha)^{2}} \frac{\sin (\pi \sqrt{n} / 2 \alpha)}{\sqrt{n}} d n-\frac{4}{(r+1) \alpha}=\frac{-4}{(r+1) \alpha},
\end{aligned}
$$

$$
\sum_{n=((4 r+7) \alpha)^{2}+1}^{((4 r+8) \alpha)^{2}} \frac{\sin (\pi \sqrt{n} / 2 \alpha)}{\sqrt{n}} \geq \int_{((4 r+7) \alpha)^{2}}^{((4 r+8) \alpha)^{2}} \frac{\sin (\pi \sqrt{n} / 2 \alpha)}{\sqrt{n}} d n=-\frac{4 \alpha}{\pi} .
$$

In view of (37)-(40), we get

$$
\begin{aligned}
& \sum_{n=\alpha^{2}+1}^{\infty} \frac{\sin (\pi \sqrt{n} / 2 \alpha)}{n \ln n} \\
& \quad \geq \sum_{r=0}\left\{\frac{4}{\pi(4 r+5) \ln ((4 r+5) \alpha)^{2}}-\frac{4}{\pi(4 r+7) \ln ((4 r+7) \alpha)^{2}}-\frac{6}{(r+1)^{2} \alpha^{2}}\right\} \\
& \geq-\frac{\pi^{2}}{\alpha^{2}} .
\end{aligned}
$$

Hence,

$$
\begin{aligned}
\sum_{n=4} \frac{1}{n \ln n} \sin \frac{\pi \sqrt{n}}{2 \alpha} & \geq \frac{1}{3 \pi \ln \alpha}-\frac{\pi^{2}+1}{\alpha^{2}}, \\
\lim _{\alpha \rightarrow \infty} \frac{f(\Delta x)}{\Delta x} & =+\infty
\end{aligned}
$$

This follows from (35) and (41).

\section{Data Availability}

No data were used to support this study. 


\section{Conflicts of Interest}

The authors declare that they do not have any conflicts of interest.

\section{References}

[1] T. W. Chaundy and A. E. Jolliffe, "The uniform convergence of a certain class of trigonometrical series," Proceedings of the London Mathematical Society, vol. s2-15, pp. 214-216, 1917.

[2] K. Duzinkiewicz and B. Szal, "On the uniform convergence of double sine series," Colloquium Mathematicum, vol. 151, no. 1, pp. 71-95, 2018.

[3] M. Dyachenko, A. Mukanov, and S. Tikhonov, "Uniform convergence of trigonometric series with general monotone coefficients," Canadian Journal of Mathematics, vol. 71, no. 6, pp. 1445-1463, 2019.

[4] M. Gabdullin, "Trigonometric series with noninteger harmonics," https://arxiv.org/abs/2102.05698.

[5] S. Kęska, "On the uniform convergence of sine series with square root," Journal of Function Spaces, vol. 2019, Article ID 1342189, 11 pages, 2019.

[6] P. Kórus, "On the uniform convergence of double sine series with generalized monotone coefficients," Periodica Mathematica Hungarica, vol. 63, no. 2, pp. 205-214, 2011.

[7] L. Leindler, "On the uniform convergence and boundedness of a certain class of sine series," Anal. Math., vol. 27, no. 4, pp. 279-285, 2001.

[8] F. Móricz, "On the uniform convergence of sine integrals," Journal of Mathematical Analysis and Applications, vol. 354, no. 1, pp. 213-219, 2009.

[9] K. A. Oganesyan, "Uniform convergence criterion for nonharmonic sine series," Sbornik, vol. 212, no. 1, pp. 70-110, 2021.

[10] I. E. Žak and A. A. Šneider, "Conditions for uniform covergence of double sine series," Izv. Vysš. Uč ebn. Zaved. Matematika, vol. 4, pp. 44-52, 1966.

[11] S. P. Zhou, P. Zhou, and D. S. Yu, "Ultimate generalization to monotonicity for uniform convergence of trigonometric series," Science China Mathematics, vol. 53, no. 7, pp. 1853$1862,2010$. 